SLAC-PUB-8745

December 2000

\title{
Experimental Issues for Precision Electroweak Physics at a High-Luminosity Z Factory
}

\author{
P. C. Rowson* and M. Woods \\ Stanford Linear Accelerator Center, Stanford, CA 94309 \\ * conference speaker
}

\begin{abstract}
We discuss the ultimate precision for $A_{L R}$, and therefore for the weak mixing angle, at a high luminosity Linear Collider. Drawing on our experience at the SLC, and considering various machine parameter sets for the NLC and for TESLA, it emerges that a compromise between peak luminosity and precision will be a likely outcome. This arises due to the severe requirements on the uncertainty in the luminosity weighted collision energy $\left(E_{C M}\right)$. We consider the cases with and without a polarized positron beam.
\end{abstract}

\section{INTRODUCTION}

The determination of the weak mixing angle $\sin ^{2} \theta_{W}^{\text {eff }}$ derived from the $A_{L R}$ measurement at the SLC is by far the most precise presently available. Based on 540 thousand events with a mean electron beam polarization of $\sim 72 \%$ the uncertainty on $A_{L R}$ consists of an approximately $1.3 \%$ statistical error, and a $0.64 \%$ systematic error (all errors are relative) leading to a precision of \pm 0.00027 on $\sin ^{2} \theta_{W}^{\text {eff }}[1]$. The measurement, now statistically limited, is the only $\sin ^{2} \theta_{W}^{\mathrm{eff}}$ determination that shows promise for sufficiently improved systematic error to be useful at a future Z factory, where data samples of order $10^{9}$ events are possible.

As is well known, the dominant systematic error is due to polarimetry ; an uncertainty of $0.50 \%$ has been obtained using a Compton polarimeter. Less well known is that the next largest systematic error $(0.39 \%)$ arises from the conversion of $A_{L R}\left(E_{c m}\right)$ to its Z-pole value $A_{L R}^{0}$ (and hence to $\sin ^{2} \theta_{W}^{\text {eff }}$ ) by correcting for initial state radiation and the contribution and interference due to the pure photon amplitude. This calculation requires accurate and precise knowledge of the luminosity-weighted average center-of-mass collision energy $E_{C M}$. A useful rule of thumb is that a $80 \mathrm{MeV}$ error in $E_{C M}$ translates into a $1 \%$ uncertainty in $A_{L R}^{0}$. From this rule, along with the fact that the statistical error $\delta A_{L R}=(\mathcal{P} \sqrt{(N)})^{-1}$ 
TABLE 1. Systematic uncertainties that affect Compton polarimetry. The chromaticity and IP corrections are due to the accelerator rather than the polarimeter.

\begin{tabular}{lcc}
\hline Uncertainty & $\mathrm{SLC} \delta \mathcal{P}_{e} / \mathcal{P}_{e}(\%)$ & Future $\mathrm{LC} \delta \mathcal{P}_{e} / \mathcal{P}_{e}(\%)$ \\
\hline Analyzing power calibration & 0.40 & 0.20 \\
Detector linearity & 0.20 & 0.10 \\
Laser polarization & 0.10 & 0.10 \\
Electronic noise & 0.20 & 0.05 \\
\hline Total polarimeter uncertainty & 0.50 & 0.25 \\
Chromaticity and IP corrections & 0.15 & negl. \\
\hline
\end{tabular}

and $A_{L R} \sim 0.15$, it is apparent that a sample of $10^{9}$ events at an effective polarization of close to $100 \%$ would require an understanding of $E_{C M}$ at the level of $1 \mathrm{MeV}$. We will return to this point following a discussion of all other systematic effects.

\section{POLARIMETRY AND POLARIZATION}

At a future Linear Collider (LC), two possibilities may be envisioned : either only the electron beam is polarized (as it was at the SLC), or a suitable positron source can be built and both beams are polarized. We will deal with these possibilites in turn. For the following discussion, we will assume that hoped for improvements in photocathode technology will provide $90 \%$ electron polarization (all results are easily scalable) [2].

In the event that only the electron beam is polarized, it is likely that a precision Compton polarimeter would be used [3]. A Compton polarimeter detects beam electrons that have been scattered by photons from a circularly polarised laser. The choice of a Compton scattering polarimeter is dictated by the requirements that the device be operated continually while beams are in collision and that uncertainties in the physics of the scattering process not be a limiting factor in the systematic error; both troublesome issues for Møller scattering instruments. In addition, the pulseto-pulse controllability of the laser target polarization (at $120 \mathrm{~Hz}$ at the SLC), as well as the high polarization (99.9\%), are additional advantages over other options. Based on our experience with the SLD Compton polarimeter, how far can this technology be pushed?

Table 1 gives a breakdown of instrumental effects, and best estimates for plausible improvements. It is assummed that a multichannel electron spectrometer supplemented by Compton gamma detectors for cross calibration is used, and that the Compton scattering IP is located downstream of the $e^{+} e^{-}$collision IP to allow for tests of collisional effects [4]. The small chromaticity effect observed at the SLC is expected to be very small in a true LC (ie, without arcs), as is any collisional depolarization [5]. We conclude that a factor of two improvement over the SLC results is achievable. Were this the limiting systematic (a likely situation), it would be possible to improve on the SLD result by a factor of 5 , a precision on $\sin ^{2} \theta_{W}^{\text {eff }}$ of 
about \pm 0.00005 . A relatively "modest" data sample of 50 million events would be sufficient in this case.

If positron polarization is available, for arguments sake at the level of $50 \%$, dramatic improvements become possible. By virtue of the fact that the effective polarization is very close to $100 \%$

$$
\mathcal{P}_{\text {eff }}=\frac{\mathcal{P}_{e}+\mathcal{P}_{p}}{1+\mathcal{P}_{e} \mathcal{P}_{p}}=96.6 \%,
$$

the fractional uncertainty on $\mathcal{P}_{\text {eff }}$ is small $-0.10 \%$ if a compton polarimeter with $0.25 \%$ precision is used for both the electron and positron beams. For this very small error, issues of non-polarimetric systematics become a serious issue as will be discussed later, but in principle $\sin ^{2} \theta_{W}^{\text {eff }}$ uncertainties approaching \pm 0.00002 are obtainable with a sample of 100 million Zs.

By using the "Blondel" scheme, whereby all four $e^{+} e^{-}$helicity configurations (LL,LR,RL,RR) are collected [6], the need for any absolute polarimetry is in principle eliminated. Typically, only $10 \%$ of the collected luminosity needs to be taken in the low cross section (LL or RR) configuration. In this technique, luminosityweighted beam polarizations are obtained directly (so that for example, any collisional effects are accounted for). Polarimeters will still be needed in order to carefully monitor left-right polarization differences for each beam at the level of $10^{-3}$. This capability was demonstrated at the SLC, where it was advantageous that the electron helicity was changed randomly pulse-to-pulse. It is not clear that helicity reversals can be performed rapidly enough at a polarized positron source. Even were they done every few minutes, larger $\mathrm{L} / \mathrm{R}$ beam asymmetries and their associated uncertainties may occur. This issue warrants more detailed study. Nevertheless, it may be possible to achieve $\sin ^{2} \theta_{W}^{\text {eff }}$ precision below \pm 0.00002 in this way, so long as all non-polarimetric uncertainties can be held to a total of less than $0.10 \%$. In what follows all relevant effects are discussed, and we will argue that energy measurement will pose the greatest challenge.

\section{OTHER SYSTEMATICS}

The measured asymmetry $A_{m}$ is related to $A_{L R}$ by the following expression which incorporates a number of small correction terms in lowest-order approximation,

$$
\begin{aligned}
A_{L R}= & \frac{A_{m}}{\left\langle\mathcal{P}_{e}\right\rangle}+\frac{1}{\left\langle\mathcal{P}_{e}\right\rangle}\left[f_{b}\left(A_{m}-A_{b}\right)-A_{\mathcal{L}}+A_{m}^{2} A_{\mathcal{P}}\right. \\
& \left.-E_{c m} \frac{\sigma^{\prime}\left(E_{c m}\right)}{\sigma\left(E_{c m}\right)} A_{E}-A_{\varepsilon}+\left\langle\mathcal{P}_{e}\right\rangle \mathcal{P}_{p}\right],
\end{aligned}
$$

where $\left\langle\mathcal{P}_{e}\right\rangle$ is the mean luminosity-weighted polarization; $f_{b}$ is the background fraction; $\sigma(E)$ is the unpolarized Z boson cross section at energy $E ; \sigma^{\prime}(E)$ is the derivative of the cross section with respect to $E ; A_{b}, A_{\mathcal{L}}, A_{\mathcal{P}}, A_{E}$, and $A_{\varepsilon}$ are the 
left-right asymmetries [7] of the residual background, the integrated luminosity, the beam polarization, the center-of-mass energy, and the product of detector acceptance and efficiency, respectively; and $\mathcal{P}_{p}$ is any longitudinal positron polarization which is assumed to have constant helicity [8].

At the SLC backgrounds were understood at the level of $3 \times 10^{-4}$. While linear colliders are inherently less forgiving than storage rings, we believe the required performance of $10^{-4}$ or better can be attained. Luminosity asymmetries $\left(A_{\mathcal{L}}\right)$ at the SLC were reduced using feedback at the source and by occasional reversals using a spin rotator solenoid, and were known to approximately $10^{-4}$. With improved small angle Bhabha and radiative Bhabaha detectors, it should be possible to do even better, if the frequency of helicity reversal for the positrons (discussed above for the case of $A_{\mathcal{P}}$ ) does not present difficulties. The other asymmetries $A_{E}$ (about $10^{-7}$ at the SLC), and particularly $A_{\varepsilon}$ [9], should not present a problem). We note that in the event positrons are nominally unpolarized, the precision expected for this case makes it necessary to verify this to better than $2 \times 10^{-4}$. At the SLC, a dedicated experiment achieved a precision of $\delta \mathcal{P}_{p}=7 \times 10^{-4}$, so this goal seems reasonable.

Finally, overlapping Z events may be a complication, in particular in the NLC design. Even at lower NLC luminosities $\left(2.6 \times 10^{33}\right.$ at a $120 \mathrm{~Hz}$ repetition rate), there is a $14 \%$ probability for 2 or more Z's in a given bunch train. Studies are needed to demonstrate that multiple $\mathrm{Z}$ events can be easily identified with the required reliability (for a $>10^{8}$ event sample, misidentification must be kept below $0.02 \%$ so as not to become a limiting uncertainty).

\section{A Energy systematics}

Anyone familiar with the heroic efforts at LEP required to achieve their spectacular energy uncertainties of order $1 \mathrm{MeV}$ (and a Z mass error of $2.1 \mathrm{MeV}$ ), will appreciate the problem faced at a future LC. The method of resonant depolarization will not be an option at a LC - rather it will be necessary to establish absolute calibration using precision spectrometers and the $\mathrm{Z}$ pole, with additional relative beam energy data coming from the acolinearity of Bhabha events. The required instrumentation, whether it involves SLC-style precision magnetic spectrometers located in the extraction lines, LEP-style BPM spectrometers, and possibly wire scanners at positions of high beam dispersion, will be very important and must be incorporated into any machine design. In addition, the machine stability and the (non-gaussian) energy distributions are less favorable at a LC.

Due to beamstrahlung effects, there is a potentially large difference between the sum of the undisrupted beam energies (pre-collision), and the luminosity-weighted $E_{C M}$. At the SLC, the effect was typically $>40 \mathrm{MeV}$ during high luminosity running, and the size of this effect was very sensitive to machine operating conditions. We have calculated beamstrahlung effects for a variety of NLC and TESLA parameter sets using the program GUINEAPIG [10]. We have already varified 
TABLE 2. Some Z-pole machines and parameter sets simulated using GUINEAPIG. The luminosity-weighted beamstrahlung energy loss corrections are given in the last row.

\begin{tabular}{lccccc}
\hline & SLC & NLC-90 & NLC-90(low) & TESLA-90 & TESLA-90(low) \\
\hline Luminosity $\left(10^{33} \mathrm{~cm}^{-2} \mathrm{~s}^{-1}\right)$ & 0.0024 & 3.9 & 0.9 & 6.5 & 1.5 \\
Repetition rate $(\mathrm{Hz})$ & 120 & 180 & 180 & 5 & 5 \\
Bunches per train & 1 & 95 & 95 & 2820 & 2820 \\
Bunch charge $\left(10^{10}\right)$ & 4.0 & 1.0 & 1.0 & 2.0 & 2.0 \\
$\gamma \epsilon_{x} / \gamma \epsilon_{y}\left(10^{-8} \mathrm{~m}-\mathrm{rad}\right)$ & $6000 / 1200$ & $400 / 6$ & $400 / 6$ & $1000 / 3$ & $1000 / 3$ \\
$\beta_{x} / \beta_{y}$ at IP $(\mathrm{mm})$ & $3.6 / 3.7$ & $10 / 0.10$ & $90 / 0.10$ & $15 / 0.4$ & $135 / 0.4$ \\
$\sigma_{z}(\mu \mathrm{m})$ & 920 & 125 & 125 & 400 & 400 \\
Lum.Wt. beamstr. E-loss $(\mathrm{MeV})$ & 49 & 125 & 18 & 44 & 1 \\
\hline
\end{tabular}

agreement to about $20 \%$ between GUINEAPIG and our energy measurement data (using energy spectrometers with $\sim 20 \mathrm{MeV}$ precision for beams in and out of collision) for the SLC. In our experience these effects were relatively unstable in time, and changed significantly as the luminosity was optimized. Depending entirely on Z-lineshape scans to incorporate all beamstrahlung effects might be unwise, as a $1 \mathrm{MeV}$ statistical error on the peak location would require a $\sim 4$ million event equivalent integrated luminosity and hence a sizeable fraction of a day even at the highest envisioned luminosities [11]. It is therefore a good strategy to minimize beamstrahlung as much as possible, with a reasonable goal of $10 \%$ relative precision on its determination.

Table 2 illustrates the mean energy loss corrections for a representative set of LC designs. In the "nominal" NLC design the effect is larger (125 MeV) than it was at the SLC (49 MeV), while the "nominal" TESLA design is more forgiving $(44 \mathrm{MeV})$. By operating at reduced bunch charge or increased horizontal beta function $\left(\beta_{x}\right)$, beamstrahlung effects can be substantially reduced, albeit at the cost of reduced luminosity. We investigated a number of scenarios for NLC and TESLA. For example, a nine-fold increase in $\beta_{x}$ will reduce the beamstrahlung energy losses to $18 \mathrm{MeV}$ and $1 \mathrm{MeV}$ respectively, with concurrent losses in luminosity by factors of 4.8 and 4.3 relative to nominal [12]. These reduced-luminosity configurations would probably be required for the highest precision $A_{L R}$ programs using 1 billion $\mathrm{Z}$ events where $\mathcal{O}(2 \mathrm{MeV})$ energy precision is needed. In general the TESLA design is more favorable for these more ambitious goals.

\section{CONCLUSIONS}

A five-fold improvement over the SLD result, to $\delta \sin ^{2} \theta_{W}^{\mathrm{eff}}= \pm 0.00005$, is plausible with a $(90 \%)$ polarized electron beam and about 50 million events. With both beams polarized $(90 \% / 50 \%)$, an error approaching \pm 0.00002 may be possible if energy uncertainties are at the $5 \mathrm{MeV}$ level. To reach this high precision will probably require a reduced-beamstrahlung/reduced-luminosity machine configuration, most easily attained in the TESLA design. In addition, the issue of rapid reversal 
of the polarized positron source, necessary for the control of systematic left-right asymmetries, requires further study.

We note that the precision electroweak program at a LC would include only modest improvements in the $\mathrm{Z}$ lineshape parameters compared to the LEP results, but might provide a $\mathrm{W}$ mass measurement to better than $10 \mathrm{MeV}\left(6 \mathrm{MeV}\right.$ for $\left.100 \mathrm{fb}^{-1}\right)$ [13]. Many of the energy related issues discussed for $A_{L R}$ would apply to a W threshold scan; in addition this measurement would require a long extrapolation from the Z-pole energy calibration point. The ultimate precision electroweak program at a LC would, we think, require somewhat specialized running conditions and reduced luminosity, either at the $\mathrm{Z}$ pole or the $\mathrm{W}$ threshold.

\section{REFERENCES}

1. K. Abe et al. [SLD Collaboration], Phys. Rev. Lett. 84, 5945 (2000).

2. A.V. Subashiev and J.E. Clendenin, SLAC-PUB-8312, (2000).

3. M. Woods, SLAC-PUB-8397, (2000).

4. A downstream Compton IP is not presently accomodated in the TESLA design. For this machine, collisional effects would need to be controlled using positron polarization and the "Blondel" scheme discussed at the end of section II.

5. P. Chen and K. Yokoya, Proceedings of the Eighth International Symposium on HighEnergy Spin Physics, Minneapolis, MN, 1988, pg. 938.

6. A. Blondel, Phys.Lett. B202,145 (1988).

7. The left-right asymmetry for a quantity $Q$ is defined as $A_{Q} \equiv\left(Q_{L}-Q_{R}\right) /\left(Q_{L}+Q_{R}\right)$ where the subscripts $L, R$ refer to the left- and right-handed beams, respectively.

8. Since the colliding electron and positron bunches were produced on different machine cycles and since the electron helicity of each cycle was chosen randomly, any positron helicity arising from the polarization of the production electrons was uncorrelated with electron helicity at the IP. However, positron polarization of constant helicity would affect the measurement.

9. The value of $A_{L R}$ is unaffected by decay-mode-dependent variations in detector acceptance and efficiency provided that the efficiency for detecting a fermion at some polar angle (with respect to the electron direction) is equal to the efficiency for detecting an antifermion at the same polar angle. Charge-sign dependent detection efficiency should be an extremely small effect in any operating or planned collider detector.

10. D. Schulte, Ph.D. thesis, Hamburg, 1996.

11. At the SLC, about $10 \mathrm{~K}$ event equivalents were used in an optimized three-point peak scan to achieve a $20 \mathrm{MeV}$ statistical error.

12. Updated NLC machine parameter sets, somewhat different then those given here, were presented by Tor Raubenheimer (see these proceedings).

13. K. Mönig, these proceedings. See also R. Hawking and K. Mönig, DESY 99-157, (1999). 